\title{
Pressure Injury due to Prolonged Face Mask Use during the COVID-19 Pandemic
}

\author{
Yousef Hammad, DMD*, Timothy W Neal, DDS, Scott R Sullivan, DDS and Thomas Schlieve, \\ $D D S, M D$
}

Division of Oral and Maxillofacial Surgery, Department of Surgery, The University of Texas Southwestern Medical Center/Parkland Memorial Hospital, Harry Hines Blvd, Dallas, TX, USA

\begin{abstract}
During the coronavirus disease 2019 (COVID-19) pandemic, the importance of wearing a mask has been stressed to the public by the center for disease control and prevention (CDC). In hospital settings, healthcare workers shifted from wearing masks during procedures and exams to all day wear. This shift has led to reports of pressure injuries and skin reactions. With prolonged face mask wear, the pressure from the elastic straps that go over and around the ears creates skin indentations that may lead to skin irritation. Healthcare workers are taught the principles of proper disposal, hygiene, and use of personal protective equipment (PPE). For the general population and especially individuals with limited access to care, these principles are not always obvious. The current report describes a 56-year-old homeless man who presented with pressure injuries of both ears from the elastic straps of his face mask due to approximately one month of uninterrupted mask wear. The patient was treated with irrigation and debridement followed by wound repair, and he healed appropriately. The purpose of this report is to shed light on a potential mechanism of injury related to excessive use of face masks in marginalized populations. Although various preventable methods for mask-related skin reactions exist and are available to many individuals, people experiencing homelessness are less likely to have access to such measures. This case aims to illustrate an extreme sequela of uninterrupted mask wear during the COVID-19 pandemic and highlight the importance of patient education regarding proper usage of PPE and personal hygiene.
\end{abstract}

\section{Introduction}

Coronavirus disease 2019 (COVID-19) has led to tremendous pressures on both foreign and domestic government officials and organizations to control the spread of the virus. Various local, regional, and global measures including border closures, school and nonessential business closures, stay-at-home orders, social distancing guidelines, and mask mandates have been deployed to slow the progression of the pandemic [1]. Laboratory studies have shown that masks may be beneficial in decreasing the spread of infections transmitted by aerosolized respiratory droplets. Some literature has shown a significantly favorable effect of public mask use on COVID-19 incidence [2-4]. A statewide mask mandate was released by the Texas governor on July 2, 2020 which required every person in Texas to wear a face covering over the nose and mouth when inside a building or space open to the public, or when in an outdoor public space wherever it is not possible to maintain six feet of social distancing. During the COVID-19 pandemic, the importance of wearing a mask use was stressed to the public by the center for disease control and prevention (CDC) in an effort to decrease the transmission of cases.

In hospital settings, healthcare workers shifted from wearing masks to perform procedures and examinations to all day wear. This shift has led to reports of pressure injuries and skin reactions related to prolonged face mask wear. One study reported a $42.8 \%$ prevalence of skin injuries caused by personal protective equipment (PPE) in medical staff during the COVID-19 pandemic [5]. With prolonged face mask wear, the pressure from the elastic straps that go over and around the ears creates skin indentations that may lead to skin irritation or pressure injuries. A pressure injury is a localized injury to the skin and underlying structures caused by prolonged pressure [6]. Extended periods of face mask use can generate excessive sweating, moisture, and friction [7]. This moisture and friction can lead to contact dermatitis

*Corresponding author: Yousef Hammad, DMD, Division of Oral and Maxillofacial Surgery, Department of Surgery, The University of Texas Southwestern Medical Center/Parkland Memorial Hospital, 5323 Harry Hines Blvd, Dallas, TX 75390, USA, Tel: 708-717-7083

Accepted: January 29, 2022

Published online: January 31, 2022

Citation: Hammad Y, Neal TW, Sullivan SR, et al. (2022) Pressure Injury due to Prolonged Face Mask Use during the COVID-19 Pandemic. J Head Neck Surg 4(1):166-169 
amongst other conditions that can have a negative impact on quality of life. In addition, prolonged pressure and friction between the mask elastic straps and ears can lead to abrasion through the skin which can become a potential nidus for skin or cartilage infection. Healthcare workers are taught the principles of proper disposal, hygiene, and use of PPE. For the general population and especially for individuals of lower income with limited access to health care and resources, the principles are not always obvious.

The current report describes a 56-year-old homeless man who presented with pressure injuries of both ears caused by the elastic straps of his face mask due to prolonged and uninterrupted mask wear. The purpose of this report is to shed light on a potential mechanism of injury related to excessive use of face masks in certain patient populations. This case aims to illustrate a truly unique case of ear trauma caused by uninterrupted mask wear during the COVID-19 pandemic.

\section{Case Report}

A 56-year-old African American male with history significant for homelessness, cocaine use disorder, hypertension, and congestive heart failure presented to the Parkland Memorial Hospital (PMH) Emergency Department (ED) in June of 2021 with a chief complaint of bilateral ear pain. Oral and maxillofacial surgery (OMS) was consulted to evaluate the patient's bilateral ear injuries. On history, it was discovered that the patient had been wearing the same surgical mask for approximately four weeks such that the elastic straps were embedded in his skin. He reported that the elastic straps had dug into his ears and that he could not remove the mask. He stated that he had been wearing the mask continuously and uninterrupted for almost one month, and over the past few weeks the elastic straps began eroding into his ears. The patient endorsed bilateral otalgia but denied any fever, chills, nausea, or vomiting.

Upon physical examination, the patient was disheveled and had bilateral ear swelling. His surgical mask was positioned around his chin with the elastic straps embedded in his skin around his ears. He had bilateral curvilinear pressure injuries measuring approximately six centimeters in length. These wounds were located at the posterior superior portion of the junction forming the crease between the pinna and the face bilaterally (Figure 1). Additionally, there was exudative crusting and scabbing of the wounds. The tympanic membranes were clear and without exudate, blood, masses or erythema bilaterally and there were no signs of perichondral hematoma. Informed consent was obtained from the patient to proceed with irrigation and debridement and wound repair under local anesthesia. The patient was prepped in a sterile fashion, and local anesthesia was achieved with fifteen milliliters of $1 \%$ lidocaine administered via field blocks of the ears bilaterally. The elastic straps of the surgical mask were then removed, and the wounds were irrigated with copious normal saline followed by careful debridement. The depths of the wounds were determined to be through epidermis, dermis, and subcutaneous tissue down to perichondrium (Figure 2). Beginning on the left side, the deep subcutaneous layer was sutured with 3-0 vicryl sutures in a simple interrupted fashion. Then, the subcuticular layer was reapproximated with 4-0 monocryl sutures in a simple interrupted fashion. Lastly, the skin was closed in a running continuous fashion with 5-0 fast gut sutures with eversion of the wound edges (Figure 3). The same steps were repeated on the contralateral ear (Figure 3).

The patient was admitted to the medicine service overnight for volume overload. Upon morning rounds, his wounds appeared to be hemostatic and without signs of infection on post-operative day one. The patient was discharged on a one-week course of antibiotics and was instructed to apply bacitracin to the wounds twice daily. He was also given wound care and scar care instructions, and he was instructed to return to our outpatient OMS clinic for follow up. He returned to our clinic two weeks later, and his bilateral ear wounds appeared to be healing well without any signs of infection or hematoma formation. The patient was instructed to remove his surgical mask rather than put it over his chin when he does not need to have it on.
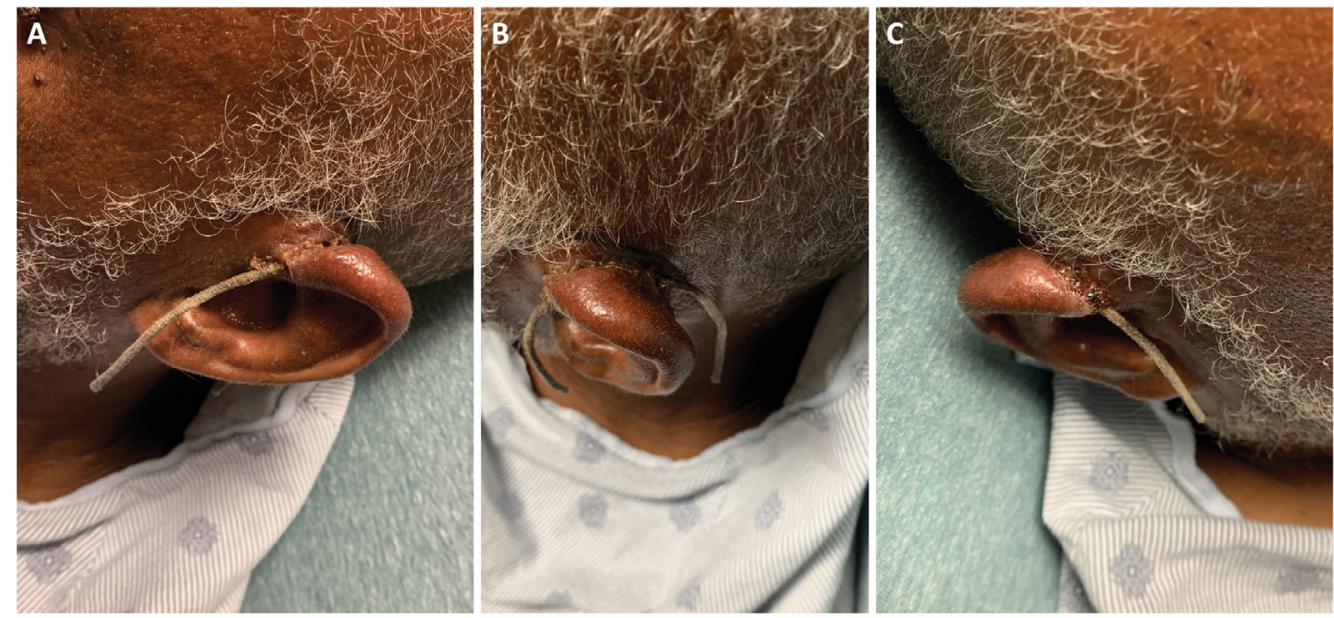

Figure 1: Pre-operative presentation of bilateral ear pressure injuries caused by the elastic straps of a surgical face mask. (A) Left ear; (B) Left ear (C) Right ear. 


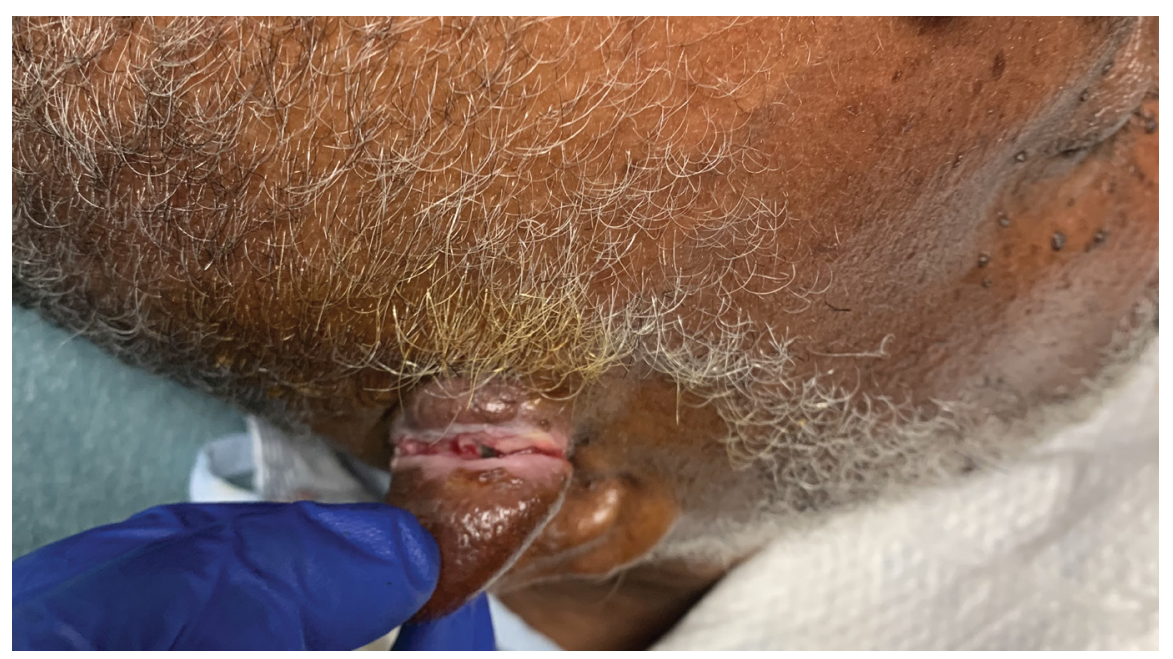

Figure 2: Intra-operative image demonstrating the depth of the right ear wound which was nearly identical to the depth of the contralateral ear.
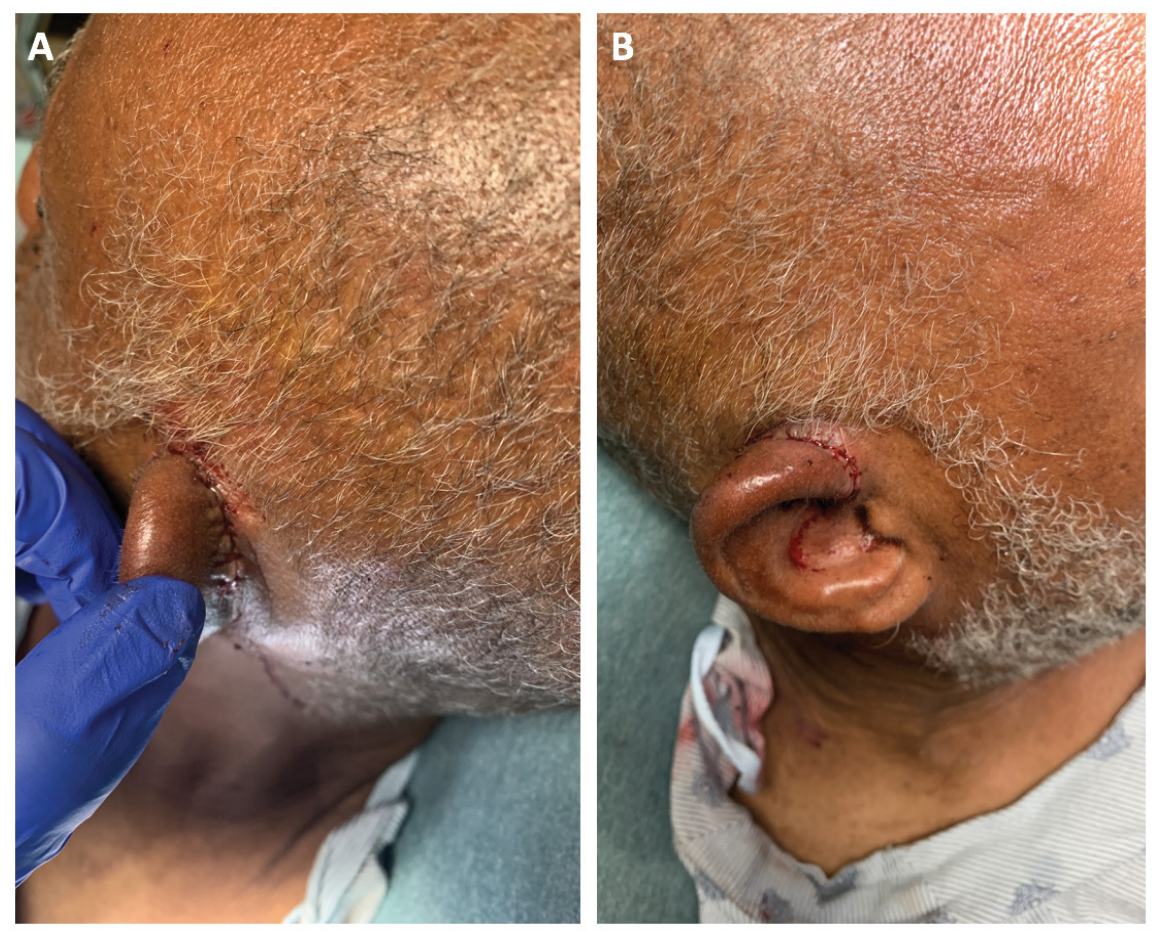

Figure 3: Post-operative images of the ear wound repairs. (A) Left ear; (B) Right ear.

\section{Discussion}

This report demonstrates an extreme case of the potential complications related to prolonged and uninterrupted mask wear. The patient described in this report was homeless and had limited access to care, but he was aware of the mask mandate in Texas that was issued in July of 2020. He was unable to obtain the COVID-19 vaccine, and he was unaware that the mask mandate in Texas had ended as of March 10, 2021. He had heightened fear because of the COVID-19 pandemic and as a result was hypervigilant in his compliance with wearing his mask. However, he was never instructed on how to properly use his mask or on how to prevent adverse skin reactions.
Various preventable measures exist for mask-related skin reactions. The simplest of which include cleansing the face and regularly replacing used masks. Additionally, avoiding humidity and hot environments as well as the prophylactic use of hydrocolloid dressings or silicone gels at pressure points on the face and ears can prevent friction injuries [8]. Patients who experience dermatitis can use moisturizers for mild presentations and topical glucocorticoid cream without fluorine for more severe cases [9]. In addition, topical antibiotic ointments such as mupirocin or bacitracin can be applied to infected skin or wounds [10].

Although these measures may be available to many individuals, the homeless and underserved populations 
are less likely to have access to such preventable methods. Individuals that experience homelessness are more likely to have difficulty adhering to public health directions including social distancing and isolation [11]. The lack of safe housing and higher prevalence of risk factors in homeless populations puts these individuals at a higher risk of severe COVID-19.

Since COVID-19 is transmitted via respiratory droplets, face masks appear to represent an effective solution to limit the spread of infection from person to person. According to the $C D C$, it is not necessary to wear a mask when outside alone and away from others or in the proximity of those living in your household. The patient in this case was overly compliant with his surgical mask wear and failed to remove his mask from his face for approximately one month which led to the development of bilateral ear pressure injuries. This case demonstrates an extreme sequela of uninterrupted mask wear during the COVID-19 pandemic and highlights the importance of patient education related to proper usage of PPE and personal hygiene. As healthcare providers, these principles are inherent. However, to certain vulnerable populations, these principles should be thoroughly explained. Future endeavors aimed at educating homeless and underserved populations on the proper usage of personal protective equipment (PPE) and personal hygiene are needed to increase awareness regarding the benefits and potential risks of chronic mask wear.

\section{Declarations of Interest}

None.

\section{References}

1. Agrawal S, Bhandari S, Bhattacharjee A, et al. (2020) City-scale agent-based simulators for the study of non-pharmaceutical interventions in the context of the Covid-19 epidemic. J Indian Inst Sci 12: 1-39.

2. Lyu W, Wehby GL (2020) Community use of face masks and Covid-19: Evidence from a natural experiment of state mandates in the US. Health Aff (Millwood) 39: 1419-1421.
3. Cheng VC, Wong S-C, Chuang VW, et al. (2020) The role of community-wide wearing of face mask for control of coronavirus disease 2019 (Covid-19) epidemic due to SARS-CoV-2. J Infect 81: 107-114.

4. Leffler CT, Ing EB, Lykins JD, et al. (2020) Association of countrywide coronavirus mortality with demographics, testing, lockdowns, and public wearing of masks. Am J Trop Med Hyg 103: 2400-2411.

5. Jiang Q, Song S, Zhou J, et al. (2020) The prevalence, characteristics, and prevention status of skin injury caused by personal protective equipment among medical staff in fighting Covid-19: A multicenter, cross-sectional study. Adv Wound Care 9: 357-364.

6. Moore Z, McEvoy NL, Avsar P, et al. (2021) Facial pressure injuries and the Covid-19 pandemic: Skin protection care to enhance staff safety in an acute hospital setting. J Wound Care 30: 0969-0700.

7. Di Altobrando A, La Placa M, Neri I, et al. (2020) Contact dermatitis due to masks and respirators during Covid-19 pandemic: What we should know and what we should do. Dermatol Ther 33: e14528.

8. Balato A, Ayala F, Bruze M, et al. (2020) European task force on contact dermatitis statement on coronavirus 19 disease (Covid-19) outbreak and the risk of adverse cutaneous reactions. J Eur Acad Dermatol Venereol 34: e353-e354.

9. Auler AC, Cássaro FAM, da Silva VO, et al. (2020) Evidence that high temperatures and intermediate relative humidity might favor the spread of Covid-19 in tropical climate: A case study for the most affected Brazilian cities. Sci Total Environ 729: 139090.

10. Yan Y, Chen H, Chen L, et al. (2020) Consensus of Chinese experts on protection of skin and mucous membrane barrier for health-care workers fighting against coronavirus disease 2019. Dermatol Ther 33: e13310.

11. Perri M, Dosani N, Hwang SW (2020) Covid-19 and people experiencing homelessness: Challenges and mitigation strategies. CMAJ 192: E716-E719.

DOI: $10.36959 / 605 / 563$

Copyright: (c) 2022 Hammad Y, et al. This is an open-access article distributed under the terms of the Creative Commons Attribution License, which permits unrestricted use, distribution, and reproduction in any medium, provided the original author and source are credited. 